This item was submitted to Loughborough's Research Repository by the author.

Items in Figshare are protected by copyright, with all rights reserved, unless otherwise indicated.

\title{
Effect of Poisson's ratio mismatch on brittle interfacial cracking between two dissimilar elastic layers
}

PLEASE CITE THE PUBLISHED VERSION

http://dx.doi.org/10.1016/j.compstruct.2016.09.024

PUBLISHER

(C) Elsevier

VERSION

AM (Accepted Manuscript)

\section{PUBLISHER STATEMENT}

This work is made available according to the conditions of the Creative Commons Attribution-NonCommercialNoDerivatives 4.0 International (CC BY-NC-ND 4.0) licence. Full details of this licence are available at: https://creativecommons.org/licenses/by-nc-nd/4.0/

\section{LICENCE}

CC BY-NC-ND 4.0

\section{REPOSITORY RECORD}

Wood, Joseph D., Christopher Harvey, and Simon Wang. 2016. "Effect of Poisson's Ratio Mismatch on Brittle Interfacial Cracking Between Two Dissimilar Elastic Layers”. figshare. https://hdl.handle.net/2134/22616. 


\title{
Effect of Poisson's ratio mismatch on brittle interfacial cracking between two dissimilar elastic layers
}

\author{
J. D. Wood, C. M. Harvey*, S. Wang \\ Department of Aeronautical and Automotive Engineering, Loughborough University, \\ Loughborough, Leicestershire LE11 3TU, United Kingdom
}

\begin{abstract}
Previous work by the authors (Harvey et al., 2015) on brittle interfacial cracking between two dissimilar elastic layers is extended to accommodate Poisson's ratio mismatch in addition to the existing capability for elastic modulus mismatch. Under crack tip bending moments and axial forces, it is now possible to use a completely analytical 2D elasticity-based theory to calculate the complex stress intensity factor (SIF) and the crack extension size-dependent energy release rates (ERRs). To achieve this, it is noted that for a given geometry and loading condition, the total ERR and bimaterial mismatch coefficient are the two main factors affecting the partitions of ERR. Based on this, equivalent material properties are derived for each layer, namely, an equivalent elastic modulus and an equivalent Poisson's ratio, such that both the total ERR and the bimaterial mismatch coefficient are maintained in an alternative equivalent case. Cases for which no analytical solution for the SIFs and ERRs currently exist can therefore be 'transformed' into cases for which the analytical solution does exist. The approach is verified against results from 2D finite element method simulations in which excellent agreement is observed for cases of plane stress and plane strain with a variety of loading conditions.
\end{abstract}

Keywords: Bimaterials; Elastic modulus mismatch; Energy release rate; Interfacial fracture; Poisson's ratio mismatch; Stress intensity factors

* Corresponding Author 


\begin{tabular}{|c|c|}
\hline \multicolumn{2}{|l|}{ Nomenclature } \\
\hline$a$ & crack length in a DCB \\
\hline$b$ & width of a DCB \\
\hline$E_{1}, E_{2}$ & Young's modulus of upper and lower beams \\
\hline$\overline{E_{1}}$ & effective Young's modulus of the upper beam \\
\hline$\tilde{E}_{1}$ & equivalent Young's modulus of the upper beam \\
\hline$\widetilde{E}_{1}$ & equivalent effective Young's modulus of the upper beam \\
\hline$G, G_{I}, G_{I I}$ & total, mode I and mode II ERRs \\
\hline$h_{1}, h_{2}, h$ & thicknesses of upper, lower and intact beams \\
\hline$K_{I}, K_{I I}$ & real and imaginary parts of the complex SIF \\
\hline$k$ & Kolosov constant \\
\hline$M_{1}, M_{2}$ & DCB tip bending moments on upper and lower beams \\
\hline$M_{1 B}, M_{2 B}, M_{B}$ & crack tip bending moments on upper, lower and intact beams \\
\hline$N_{1}, N_{2}$ & DCB tip axial forces on upper and lower beams \\
\hline$N_{1 B}, N_{2 B}, N_{B}$ & crack tip axial forces on upper, lower and intact beams \\
\hline$r$ & radius coordinate centered on crack tip \\
\hline$\gamma$ & thickness ratio, $\gamma=h_{2} / h_{1}$ \\
\hline$\delta a$ & crack extension size \\
\hline$\varepsilon$ & bimaterial mismatch coefficient \\
\hline$\eta$ & Young's modulus ratio, $\eta=E_{2} / E_{1}$ \\
\hline $\bar{\eta}$ & effective Young's modulus ratio \\
\hline$\tilde{\eta}$ & equivalent Young's modulus ratio \\
\hline$\mu_{1}, \mu_{2}$ & shear modulus of upper and lower beams \\
\hline $\mathrm{N}$ & ratio of Poisson's ratios, $\mathrm{N}=v_{2} / v_{1}$ \\
\hline$v_{1}, v_{2}$ & Poisson's ratio of upper and lower beams \\
\hline$\tilde{v}$ & equivalent Poisson's ratio, $v_{1}=v_{2}=\tilde{v}$ \\
\hline$\sigma_{n}, \tau_{s}$ & interfacial opening stress and shear stress \\
\hline DCB & double cantilever beam \\
\hline ERR & energy release rate \\
\hline FEM & finite element method \\
\hline SIF & stress intensity factor \\
\hline
\end{tabular}

\section{Introduction}

It is well known from the work of Williams [1] that the stress intensity factor (SIF) for a brittle interfacial crack between two dissimilar elastic layers is of complex form, that is, $K=K_{I}+i K_{I I}$. The complex SIF indicates oscillatory singularities in the elastic field around the crack tip. This was shown in 1959 and since then one of the major challenges in the field of 
fracture mechanics has been to analytically obtain the SIFs, $K_{I}$ and $K_{I I}$, and the crack extension size-dependent energy release rate (ERR) components, $G_{I}$ and $G_{I I}$.

Harvey et al. [2] have recently established a completely analytical theory to calculate $K_{I}, K_{I I}$ , $G_{I}$ and $G_{I I}$ for a brittle interfacial crack between two elastic materials with an elastic modulus mismatch but with equal Poisson's ratios, that is, with $E_{1} \neq E_{2}$ and $v_{1}=v_{2}$, under bending moments and axial forces (subscripts 1 and 2 represent the upper and lower layers respectively). The theory [2] was extensively verified in Ref. [3]. A limitation of the theory [2] is that the Poisson's ratio of each layer must be the same, that is, $v_{1}=v_{2}$. In applications of layered material systems, for example in thermal barrier coatings in gas turbine engines or in surface coatings to protect against corrosion, friction and wear, it is typical, however, to have a mismatch in the Poisson's ratio as well as in the elastic modulus. It is therefore important that the theory [2] is extended to accommodate Poisson's ratio mismatch in addition to the existing capability for elastic modulus mismatch, that is, to accommodate both $E_{1} \neq E_{2}$ and $v_{1} \neq v_{2}$. This paper reports such an extension.

To achieve this extension, it is noted that for a given geometry and loading condition, the total ERR and the bimaterial mismatch coefficient (the oscillation index of the interfacial stresses) are the two main factors affecting the partitions of ERR. Based on this, the approach has been to derive equivalent material properties for each layer, namely, an equivalent elastic modulus and an equivalent Poisson's ratio, such that both the total ERR and the bimaterial mismatch coefficient are maintained in an alternative case. Cases for which no analytical solution for the SIFs and ERRs currently exist can therefore be 'transformed' into other cases for which the analytical solution does exist [2].

This paper is organised as follows: In Section 2, equivalent material properties are derived so that cases with $v_{1} \neq v_{2}$ can be used with the analytical partition theory in Ref. [2]. In Section 3, the approach is verified against results from 2D finite element method (FEM) simulations with $v_{1} \neq v_{2}$ and with a variety of loading conditions. Finally conclusions are drawn in Section 4. Note that this paper is a supplement to work previously published by the authors in Refs. [2,3]. Due to the complexity of the previous work [2,3], only the new analytical development is presented here with accompanying background only where necessary. The reader is directed to Refs. [2,3] for further information and full details. A review of the literature can also be found in Ref. [2]. 


\section{Analytical development}

\subsection{Interfacial stresses ahead of the crack tip}

Fig. 1a shows a bimaterial double cantilever beam (DCB) with its geometry, tip bending moments, $M_{1}$ and $M_{2}$, and tip axial forces, $N_{1}$ and $N_{2}$. The Young's modulus, shear modulus and Poisson's ratio of beam $i$ are denoted by $E_{i}, \mu_{i}$ and $v_{i}$ respectively (with $i=1,2$ ). The interfacial opening stress and shear stress ahead of the crack tip, $\sigma_{n}$ and $\tau_{s}$, can be expressed in combined complex form as [4]

$$
\sigma_{n}+i \tau_{s}=\frac{\left(K_{I}+i K_{I I}\right)}{\sqrt{2 \pi r}} r^{i \varepsilon}
$$

or in individual real form as

$$
\begin{aligned}
& \sigma_{n}=\frac{1}{\sqrt{2 \pi r}}\left\{K_{I} \cos [\varepsilon \ln (r)]-K_{I I} \sin [\varepsilon \ln (r)]\right\} \\
& \tau_{s}=\frac{1}{\sqrt{2 \pi r}}\left\{K_{I} \sin [\varepsilon \ln (r)]+K_{I I} \cos [\varepsilon \ln (r)]\right\}
\end{aligned}
$$

where $r$ is the radius coordinate centered on the crack tip and $K_{I}$ and $K_{I I}$ are the real and imaginary parts of the complex SIF. The signs of $\sigma_{n}$ and $\tau_{s}$ are positive in the directions shown in Fig. 1b. In Eqs. (1) to (3), the bimaterial mismatch coefficient $\varepsilon$ is defined as

$$
\varepsilon=\frac{1}{2 \pi} \ln \left[\left(\frac{k_{1}}{\mu_{1}}+\frac{1}{\mu_{2}}\right)\left(\frac{k_{2}}{\mu_{2}}+\frac{1}{\mu_{1}}\right)^{-1}\right]
$$

where the Kolosov constant $k_{i}$ (with $i=1,2$ ) is defined as $k_{i}=3-4 v_{i}$ for plane strain and as $k_{i}=\left(3-v_{i}\right) /\left(1+v_{i}\right)$ for plane stress. By introducing the Young's modulus ratio, $\eta=E_{2} / E_{1}$, then $\varepsilon$ becomes

$$
\varepsilon= \begin{cases}\frac{1}{2 \pi} \ln \left[\frac{3 \eta+v_{2}-\eta v_{1}+1}{\eta-v_{2}+\eta v_{1}+3}\right] & \text { for plane stress } \\ \frac{1}{2 \pi} \ln \left[\frac{-4 \eta v_{1}^{2}+3 \eta+v_{2}-\eta v_{1}+1}{-4 v_{2}^{2}+\eta-v_{2}+\eta v_{1}+3}\right] & \text { for plane strain }\end{cases}
$$




\subsection{Total energy release rate}

From the authors previous work [2,5-11], and with reference to Fig. 1b, the total ERR $G$ of a bimaterial DCB with two crack tip bending moments, $M_{1 B}$ and $M_{2 B}$, and two crack tip axial forces, $N_{1 B}$ and $N_{2 B}$, is given by

$$
G=\frac{1}{2 \bar{E}_{1} b^{2} \bar{C}}\left\{\begin{array}{c}
M_{1 B} \\
M_{2 B} \\
N_{1 B} \\
N_{2 B}
\end{array}\right\}^{T}\left[\begin{array}{llll}
C_{11} & C_{12} & C_{13} & C_{14} \\
C_{12} & C_{22} & C_{23} & C_{24} \\
C_{13} & C_{23} & C_{33} & C_{34} \\
C_{14} & C_{24} & C_{34} & C_{44}
\end{array}\right]\left\{\begin{array}{c}
M_{1 B} \\
M_{2 B} \\
N_{1 B} \\
N_{2 B}
\end{array}\right\}=\frac{1}{2 \bar{E}_{1} b^{2} \bar{C}}\left\{\begin{array}{c}
M_{1 B} \\
M_{2 B} \\
N_{1 B} \\
N_{2 B}
\end{array}\right]^{T}[C]\left\{\begin{array}{c}
M_{1 B} \\
M_{2 B} \\
N_{1 B} \\
N_{2 B}
\end{array}\right\}
$$

where

$$
\bar{C}=\bar{\eta}^{2} \gamma^{4}+4 \bar{\eta} \gamma^{3}+6 \bar{\eta} \gamma^{2}+4 \bar{\eta} \gamma+1
$$

and where $\bar{E}_{1}$ is the effective Young's modulus of the upper beam and $\bar{\eta}$ is the effective Young's modulus ratio. For plane stress $\bar{E}_{1}=E_{1}$ and $\bar{\eta}=\eta$; for plane strain $\bar{E}_{1}=E_{1} /\left(1-v_{1}^{2}\right)$ and $\bar{\eta}=\eta\left(1-v_{1}^{2}\right) /\left(1-v_{2}^{2}\right)$. The coefficient matrix $[C]$ is given by

$$
\begin{gathered}
C_{11}=12 \bar{\eta} \gamma\left(\bar{\eta} \gamma^{3}+4 \gamma^{2}+6 \gamma+3\right) / h_{1}^{3} \\
C_{12}=-12(\bar{\eta} \gamma+1) / h_{1}^{3} \\
C_{13}=6 \bar{\eta} \gamma(\gamma+1) / h_{1}^{2} \\
C_{14}=-6(\gamma+1) / h_{1}^{2} \\
C_{22}=12\left(3 \bar{\eta} \gamma^{3}+6 \bar{\eta} \gamma^{2}+4 \bar{\eta} \gamma+1\right) /\left(\bar{\eta} h_{1}^{3} \gamma^{3}\right) \\
C_{23}=6 \bar{\eta} \gamma(\gamma+1) / h_{1}^{2} \\
C_{24}=-6(\gamma+1) / h_{1}^{2} \\
C_{33}=\bar{\eta} \gamma\left(\bar{\eta} \gamma^{3}+1\right) / h_{1} \\
C_{34}=-\left(\bar{\eta} \gamma^{3}+1\right) / h_{1} \\
C_{44}=\left(\bar{\eta} \gamma^{3}+1\right) /\left(\bar{\eta} h_{1} \gamma\right)
\end{gathered}
$$




\subsection{Equivalent bimaterial problems}

From the previous work in Ref. [2], it is observed that for a given geometry and loading condition, the bimaterial mismatch coefficient $\varepsilon$ and the total ERR $G$ are the two main factors affecting the partitions of ERR, $G_{I}$ and $G_{I I}$. Eqs. (5) and (6) show that $\varepsilon$ and $G$ depend on $E_{1}$, $\eta, v_{1}$ and $v_{2}$. It is therefore proposed that a given real case with $E_{1}, E_{2}=\eta E_{1}, v_{1}$ and $v_{2}$ can be replaced by an equivalent case with $\widetilde{E}_{1}, \widetilde{E}_{2}=\tilde{\eta} \widetilde{E}_{1}$ and $\tilde{v}_{1}=\tilde{v}_{2}=\tilde{v}$ that maintains $\varepsilon$ and $G$ with similar partitions of ERR, $G_{I}$ and $G_{I I}$. Such behavior would be advantageous to transform cases for which no analytical solution for the SIFs and ERRs currently exists into other cases for which the analytical solution does exist, such as for those in Ref. [2]. To maintain the same $\varepsilon$, the equivalent Poisson's ratio $\tilde{v}$ is obtained by using Eq. (5), as follows:

$$
\tilde{v}= \begin{cases}\frac{v_{1}+\tilde{\eta} v_{1}-2}{\tilde{\eta}-1}-\frac{(\tilde{\eta}+1)\left(v_{1}+v_{2}-2\right)}{(\eta+1)(\tilde{\eta}-1)} & \text { for plane stress } \\ \frac{2 \eta-2 \tilde{\eta}+v_{2}-\eta v_{1}+\tilde{\eta} v_{2}-3 \eta v_{1}^{2}+3 \tilde{\eta} v_{2}^{2}+v_{2}^{2}-\eta \tilde{\eta} v_{1}-\eta \tilde{\eta} v_{1}^{2}}{3 \eta-3 \tilde{\eta}+v_{2}-\eta \tilde{\eta}-\eta v_{1}+\tilde{\eta} v_{2}-4 \eta v_{1}^{2}+4 \tilde{\eta} v_{2}^{2}-\eta \tilde{\eta} v_{1}+1} & \text { for plane strain }\end{cases}
$$

It is seen from Eq. (6) that under plane stress conditions the total ERR $G$ is independent of the Poisson's ratios. Therefore, changing the values of the Poisson's ratios only affects the value of the bimaterial mismatch coefficient $\varepsilon$ and hence the partitions of the ERR, $G_{I}$ and $G_{I I}$. For this case, as the total $\operatorname{ERR} G$ is maintained regardless of $v_{1}$ and $v_{2}$, only an equivalent Poisson's ratio $\tilde{v}$ is needed and it is possible to set $\tilde{\eta}=\eta$, in which case Eq. (18) for plane stress reduces to

$$
\tilde{v}=\frac{\left(\eta v_{1}-v_{2}\right)}{(\eta-1)}
$$

In Eq. (19), if $\eta \rightarrow 1$ then $\tilde{v} \rightarrow \infty$, which is unacceptable behavior for $\tilde{v}$. Therefore when $\eta$ is close to 1 , setting $\tilde{\eta}=\eta$ is no longer suitable and Eq. (18) must be used instead with an alternative $\tilde{\eta}$.

Using an alternative $\tilde{\eta}$ affects the total ERR $G$ under both plane stress and plane strain conditions. Therefore, to maintain $G, \bar{E}_{1}$ must also be replaced by $\widetilde{\bar{E}}_{1}$, which represents the equivalent effective Young's modulus of the upper beam. Let $[\tilde{C}]$ and $\widetilde{\bar{C}}$ denote the $[C]$ and $\bar{C}$ in Eqs. (6) and (7) respectively with the substitution $\bar{\eta}=\tilde{\eta}$. To maintain the same $G,{\widetilde{E_{1}}}_{1}$ is obtained by using Eq. (6), as follows: 


$$
{\widetilde{E_{1}}}_{1}=\frac{\overline{E_{1}} \bar{C}}{\widetilde{\widetilde{C}}} \frac{\left.\left\{\begin{array}{llll}
M_{1 B} & M_{2 B} & N_{1 B} & N_{2 B}
\end{array}\right\}[\tilde{C}]\right\}\left\{\begin{array}{lllll}
M_{1 B} & M_{2 B} & N_{1 B} & N_{2 B}
\end{array}\right\}^{T}}{\left\{\begin{array}{llll}
M_{1 B} & M_{2 B} & N_{1 B} & N_{2 B}
\end{array}\right\}[C]\left[\begin{array}{llll}
M_{1 B} & M_{2 B} & N_{1 B} & N_{2 B}
\end{array}\right\}^{T}}
$$

Then, $E_{1}$ is replaced by $\widetilde{E}_{1}$ where for plane stress $\widetilde{E}_{1}=\widetilde{\bar{E}}_{1}$ and for plane strain $\widetilde{E}_{1}=\widetilde{\bar{E}}_{1}\left(1-\tilde{v}^{2}\right)$. Note that for plane stress cases with $\tilde{\eta}=\eta$, Eq. (20) reduces to $\tilde{E}_{1}=E_{1}$.

The method above derives formulae for $\tilde{v}$ and $\tilde{E}_{1}$, which are dependent on the initial selection of $\tilde{\eta}$. Any consistent combination of $\tilde{E}_{1}, \tilde{\eta}, \tilde{v}$ will maintain both $G$ and $\varepsilon$ in an alternative equivalent case with $E_{1} \neq E_{2}$ and $v_{1}=v_{2}$. The following recommends which combinations give the most accurate partitions of ERR, $G_{I}$ and $G_{I I}$. The general principle in the following is to minimize the difference between the real material properties and the equivalent material properties while still achieving $\tilde{v}_{1}=\tilde{v}_{2}=\tilde{v}$.

Based on the FEM results in Section 3, for plane stress conditions, using $\tilde{\eta}=\eta$ provides accurate results for almost the whole range of $\eta$; however, when $-0.1<\log _{10}(1 / \eta)<0.1$, since $\tilde{v} \rightarrow \infty$ as $\eta \rightarrow 1$, it has been identified that using $\tilde{\eta}=1.1$ and $\widetilde{\bar{E}}_{1}$ as given by Eq. (20) instead works well throughout this range.

For plane strain conditions, selecting the equivalent material properties, $\tilde{E}_{1}, \tilde{\eta}$ and $\tilde{v}$, is more involved as $\tilde{E}_{1}$ and $\tilde{v}$ are very sensitive to the chosen value of $\tilde{\eta}$. Initially the value of $\log _{10}(1 / \tilde{\eta})$ is varied by increments of 0.1 in the range $-2 \leq \log _{10}(1 / \tilde{\eta}) \leq 2$. If the corresponding value of $\tilde{v}$ is in the range of physically admissible Poisson's ratios, that is, $0<\tilde{v}<0.5$, then the values are saved. If only one value of $\tilde{v}$ is in this range then it is selected with the corresponding value of $\tilde{\eta}$; however, if multiple values of $\tilde{v}$ obey this condition, then the ones which minimize the arithmetic difference between $\eta$ and $\tilde{\eta}$ are selected. Finally, the value of $\tilde{E}_{1}$ can be calculated using $\widetilde{\bar{E}}_{1}$ from Eq. (20).

\section{Numerical verification}

A method has been described in Section 2 for reducing cases of bimaterial interfacial cracking with $E_{1} \neq E_{2}$ and $v_{1} \neq v_{2}$ to equivalent cases with $E_{1} \neq E_{2}$ and $v_{1}=v_{2}$. The ERRs, $G_{I}$ and $G_{I I}$, can then be calculated by using the analytical mixed-mode partition theory in Ref. [2] for brittle interfacial cracks between two elastic materials with $E_{1} \neq E_{2}$ and $v_{1}=v_{2}$. In order to verify this approach, a series of 2D FEM simulations are conducted using MSC/NASTRAN on the DCB 
shown in Fig. 1 with a range of values of $E_{1}, E_{2}, v_{1}$ and $v_{2}$, from which the ERRs, $G_{I}$ and $G_{I I}$, are calculated. The verification is then performed by comparing values of the total ERR $G$ and the ERR partition $G_{I} / G$ from the FEM and the analytical theory.

In the FEM simulations, the thickness ratio $\gamma=h_{2} / h_{1}$ is kept at a constant value of $\gamma=1$; the Young's modulus ratio $\eta=E_{2} / E_{1}$ is varied in the range $1 / 100 \leq \eta \leq 100$; the ratio of Poisson's ratios $\mathrm{N}=v_{2} / v_{1}$ is varied in the range $-0.7 \leq \log _{10}(1 / \mathrm{N}) \leq 0.7$; and the DCB tip loads are varied in the range $-10,000 \leq M_{2}, N_{1} \leq 10,000$ with $M_{1}=1000$. Note that it is not necessary to vary the thickness ratio $\gamma$. The effect of the through-thickness location of the crack on the ERRs and SIFs has already been thoroughly dealt with in Refs. [2,3]. This paper adds additional material mismatch capability, and further consideration of the thickness ratio $\gamma$ is therefore not needed. It can, however, be easily shown that the same conclusions apply if $\gamma \neq 1$. Therefore, the entire practically useful domain of cracking between bimaterial layers is considered. As the FEM is dimensionless in nature, the model's parameters are given here without units; however, if engineering scale-appropriate units are required then units of $\mathrm{mm}$ and $\mathrm{N}$ may be chosen for length and force respectively, from which the consistent set of units follows. The upper and lower beams of the DCB are modelled using quadrilateral plane stress or plane strain shell elements with a thickness of $b=10$ and isotropic material properties within each beam. The thicknesses of the upper and lower beams are equal with $h_{1}=h_{2}=1$. The minimum Young's modulus is $E_{\min }=1000$. If the modulus ratio $\eta>1$, then the Young's modulus of the upper and lower beams is selected to be $E_{1}=E_{\min }$ and $E_{2}=\eta E_{\min }$ respectively, otherwise $E_{2}=E_{\min }$ and $E_{1}=E_{\min } / \eta$. The Poisson's ratios are controlled by specifying a mean value of $\bar{v}=0.29$ such that $\bar{v}=\left(v_{1}+v_{2}\right) / 2$. The Poisson's ratio of the upper beam and lower beams are then determined as $v_{1}=2 \bar{v} /(1+\mathrm{N})$ and $v_{2}=\mathrm{N} v_{1}$ for the upper and lower beams respectively. This provided an even spread of the Poisson's ratios to be considered while still keeping the maximum value below 0.5 . Note that a value of $\mathrm{N}=1$ corresponds to no Poisson's ratio mismatch or $v_{1}=v_{2}$, as previously considered in Refs. [2,3]. The shear modulus is calculated using $\mu_{i}=E_{i} /\left[2\left(1+v_{i}\right)\right]$ with $i=1,2$ for the upper and lower beams, respectively. The uncracked length of the DCB is 100 and the cracked length is 10.

The partitions of ERR, $G_{I}$ and $G_{I I}$, depend on the crack extension size $\delta a$. The analytical partition theory in Ref. [2] accommodates any value of $\delta a$ by determining $G_{I}$ and $G_{I I}$ for 
$\delta a=0.05$, from which the SIFs, $K_{I}$ and $K_{I I}$ are determined. With knowledge of $K_{I}$ and $K_{I I}$, $G_{I}$ and $G_{I I}$ can be determined for any value of $\delta a$. In this work, therefore, the selection of $\delta a$ is somewhat arbitrary; however, if $\delta a \neq 0.05$ then the verification is even more rigorous due to the extra steps in the analytical calculation, the necessary accurate calculation of the SIFs as part of the process, and the opportunity for compounding inaccuracy. A crack extension size $\delta a=0.01$ is therefore selected in order to calculate the ERR. The choice of $\delta a$ determined the size of the elements surrounding the crack tip. Since $\delta a$ is very small ( $\delta a<<a)$, a non-uniform mesh is used in order to avoid excessive computation. 2000 square elements of size $p \times p$ are centered on the crack tip in the $x$-direction with $p=\delta a=0.01$, and 100 square elements are centered on the crack tip in the $y$-direction. Beyond the region of uniform element size surrounding the crack tip, elements are allowed to grow at a constant rate of 1.1 in both the $x$ - and $y$-directions. In the $x$ direction, the maximum element size is limited to 1.0 (no limit is needed in the $y$-direction due to the thin layers). Very small adjustments are made to the element size growth rate vertically and to the maximum element size horizontally to satisfy the boundary geometry. Axial forces, $N_{1}$ and $N_{2}$, are applied as point forces to the tips of the upper and lower beams respectively and are uniformly-distributed by area. Bending moments, $M_{1}$ and $M_{2}$, are applied as equal and opposite axial forces to the top and bottom corners of each of the upper and lower beam tips respectively. A rigid interface between the upper and lower beams is modelled by 'connecting' the translational degrees of freedom of co-located interface nodes on the upper and lower beams using multi-point constraints; however, at the crack tip, instead of rigidly connecting the crack tip nodes, the interface is modelled with normal and shear point springs. Using springs 'captures' the crack tip opening force and shearing force. The stiffness of both springs is $k_{s}=b p E_{C T}$ where $E_{C T}=10^{10}$, which is the Young's modulus of the interface at the crack tip. This meant that the spring stiffness $k_{s}$ is sufficiently high with respect to $E_{1}$ and $E_{2}$ to simulate brittle interfacial cracking without introducing excessive numerical error. Because the interface is rigid, the ERRs are calculated using the virtual crack closure technique. Contact between the upper and lower surfaces of the crack is not considered.

\subsection{Bending moments only}

The DCB is subjected to tip bending moments in order to vary the crack tip bending moment on the lower beam $M_{2 B}$ in the range $-10,000 \leq M_{2 B} \leq 10,000$ while keeping the crack tip 
bending moment on the upper beam constant at $M_{1 B}=1000$. Results from the plane stress condition are shown in Fig. 2 and results from the plane strain condition are shown in Fig. 3. Figs. 2a and 3a show the difference between the total ERR $G$ from the present theory $G_{\text {th }}$ and from the FEM $G_{\mathrm{FEM}}$, defined as $\left|1-G_{\mathrm{th}} / G_{\mathrm{FEM}}\right|$. Figs. 2b and 3b show the difference between the ERR partition $G_{I} / G$ from the present theory $\left(G_{I} / G\right)_{\text {th }}$ and from the FEM $\left(G_{I} / G\right)_{\text {FEM }}$, defined as $\left|\left(G_{I} / G\right)_{\text {th }}-\left(G_{I} / G\right)_{\mathrm{FEM}}\right|$. Note that, as described above, the present theory combines the partition theory in Ref. [2] with the method in Section 2 for transforming cases with Poisson's ratio mismatch into alternative cases with no Poisson's ratio mismatch.

It is seen from Figs. 2a (plane stress) and 3a (plane strain) that there is virtually exact agreement over the whole domain between the present theory and the FEM when considering total ERR $G$. Then, from Figs. 2b (plane stress) and 3b (plane strain), there is generally excellent agreement between the theory and the FEM when considering the ERR partition $G_{I} / G$ . In both cases, the majority of the theoretical results are within about $4 \%$ of that obtained from the FEM. From Fig. 2b (plane stress), the maximum error between the ERR partitions is 10.5\% and located at $\log _{10}(1 / \eta)=1.7, \log _{10}(1 / \mathrm{N})=0.7$ and $M_{2 B} / M_{1 B}=0$. (The error color bar has been capped at 0.10 for clear presentation.) For Fig. 3b (plane strain) the maximum error between the ERR partitions is $36.8 \%$, located at $\log _{10}(1 / \eta)=-1.2, \log _{10}(1 / \mathrm{N})=0.7$ and $M_{2 B} / M_{1 B}=5$, and rapidly diminishes.

As explained in Section 2.3, an equivalent Poisson's ratio $\tilde{v}$, an equivalent Young's modulus ratio $\tilde{\eta}$, and an equivalent Young's modulus of the upper beam $\widetilde{E}_{1}$ are all needed in order to find a suitable equivalent bimaterial case under plane strain conditions. For the selected value of $\tilde{\eta}$, two approximations are needed to find $\tilde{v}$ and $\widetilde{E}_{1}$ using Eqs. (18) and (20) respectively. Furthermore, $\tilde{v}$ and $\tilde{E}_{1}$ are very sensitive to the chosen value of $\tilde{\eta}$. In contrast, only $\tilde{v}$ is required for plane stress conditions (unless $\eta \rightarrow 1$ ), which requires only one approximation using Eq. (18) with $\tilde{\eta}=\eta$ being maintained. The increased maximum error in the plane strain results is attributed to the compounding error from the two approximations for $\tilde{v}$ and $\widetilde{E}_{1}$, while the plane stress results agree with FEM results more closely due to there being only one approximation for $\tilde{v}$ 


\subsection{Bending moments and axial forces}

The DCB is also subjected to tip axial forces and bending moments in order to vary the crack tip axial force on the upper beam $N_{1 B}$ in the range $-10,000 \leq N_{1 B} \leq 10,000$ while keeping the crack tip bending moment on the upper beam constant at $M_{1 B}=1000$. Results from the plane stress condition are shown in Fig. 4 and results from the plane strain condition are shown in Fig. 5. Figs. 4a and 5a show the difference between the total ERR $G$ from the present theory $G_{\text {th }}$ and from the FEM $G_{\mathrm{FEM}}$, defined as $\left|1-G_{\mathrm{th}} / G_{\mathrm{FEM}}\right|$. Figs. 4b and 5b show the difference between the ERR partition $G_{I} / G$ from the present theory $\left(G_{I} / G\right)_{\text {th }}$ and from the FEM $\left(G_{I} / G\right)_{\text {FEM }}$, defined as $\left|\left(G_{I} / G\right)_{\mathrm{th}}-\left(G_{I} / G\right)_{\mathrm{FEM}}\right|$.

Again it is seen from Figs. 4a (plane stress) and 5a (plane strain) that there is virtually exact agreement over the whole domain between the present theory and the FEM when considering the total ERR $G$. Then, from Figs. 4b (plane stress) and 5b (plane strain), there is also excellent agreement between the theory and the FEM when considering the ERR partition $G_{I} / G$. In both cases, the majority of the theoretical results are again within about $4 \%$ of that obtained from the FEM. For Fig. 4b (plane stress) the maximum error between the ERR partitions is $16.3 \%$, located at $\log _{10}(1 / \eta)=1.6, \log _{10}(1 / \mathrm{N})=0.4$ and $N_{1 B} / M_{1 B}=-10$, and rapidly diminishes. For Fig. $5 \mathrm{~b}$ (plane strain) the maximum error between the ERR partitions is $36.3 \%$, located at $\log _{10}(1 / \eta)=1.2, \log _{10}(1 / \mathrm{N})=-0.7$ and $N_{1 B} / M_{1 B}=10$, and rapidly diminishes.

\section{Conclusions}

The authors' existing analytical partition theory [2] for brittle interfacial cracking between two dissimilar elastic layers has been successfully extended to accommodate Poisson's ratio mismatch between the upper and lower beams in addition to its existing capability for elastic modulus mismatch. This is achieved by developing a method to transform cases with Poisson's ratio mismatch into alternative cases with no Poisson's ratio mismatch. Results for total ERR $G$ and the ERR partition $G_{I} / G$ are obtained by combining this method with the existing analytical partition theory in Ref. [2], and are compared to those obtained from the 2D FEM in order to verify the approach. Excellent agreement is observed for both cases of plane stress and plane strain and under a variety of loading conditions. Under crack tip bending moments and axial forces, it is now possible to calculate the crack extension size-dependent ERRs, $G_{I}$ and $G_{I I}$, for 
a brittle interfacial crack between two dissimilar elastic layers with a Poisson's ratio mismatch as well as a Young's modulus mismatch.

It should be remembered that this work represents an approximate method. It will be useful for researchers and engineers to quickly obtain predictions of the fracture mode partition without full FEM simulations. Despite it being an approximate method, in the majority of cases the partition can be predicted to within 4\% of the FEM result. If improved accuracy is required then it may be that only a full FEM simulation can provide this. To be confident of avoiding any of the localized areas of increased error, it is suggested to be cautious when dealing with extreme cases of Poisson's ratio mismatch, for example, $\log _{10}(1 / \mathrm{N}) \approx \pm 0.7$ (or $\mathrm{N} \approx 5^{ \pm 1}$ ).

\section{References}

[1] Williams ML. The stresses around a fault or crack in dissimilar media. Bulletin of the Seismological Society of America 1959;49:199-204.

[2] Harvey CM, Wood JD, Wang S. Brittle interfacial cracking between two dissimilar elastic layers: Part 1 - Analytical development. Composite Structures 2015;134;1076-1086.

[3] Harvey CM, Wood JD, Wang S. Brittle interfacial cracking between two dissimilar elastic layers: Part 2 - Numerical verification. Composite Structures 2015;134;1087-1094.

[4] Suo Z, Hutchinson JW. Interface crack between two elastic layers. International Journal of Fracture 1990;43:1-18.

[5] Harvey CM. Mixed-mode partition theories for one-dimensional fracture. PhD Thesis. March 2012, Loughborough University, UK.

[6] Wang S, Harvey CM. A theory of one-dimensional fracture. Composite Structures 2012;94:758-767. Also a plenary lecture at the 16th International Conference on Composite Structures (ICCS16), 28-30 June 2011, Porto, Portugal.

[7] Harvey CM, Wang S. Experimental assessment of mixed-mode partition theories. Composite Structures 2012;94:2057-2067.

[8] Wang S, Harvey CM. Mixed mode partition theories for one dimensional fracture. Engineering Fracture Mechanics 2012;79:329-352.

[9] Harvey CM, Wang S. Mixed-mode partition theories for one-dimensional delamination in laminated composite beams. Engineering Fracture Mechanics 2012;96:737-759. 
[10] Harvey CM, Wood JD, Wang S, Watson A. A novel method for the partition of mixedmode fractures in 2D elastic laminated unidirectional composite beams. Composite Structures 2014;116:589-594.

[11] Harvey CM, Eplett MR, Wang S. Experimental assessment of mixed-mode partition theories for generally laminated composite beams. Composite Structures 2015;124:10-18. 


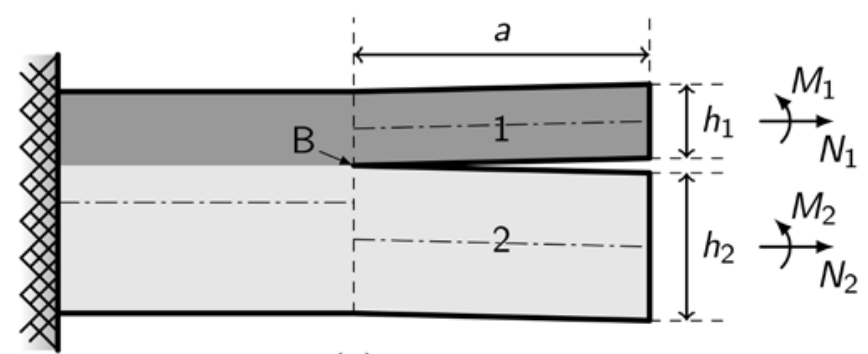

(a)

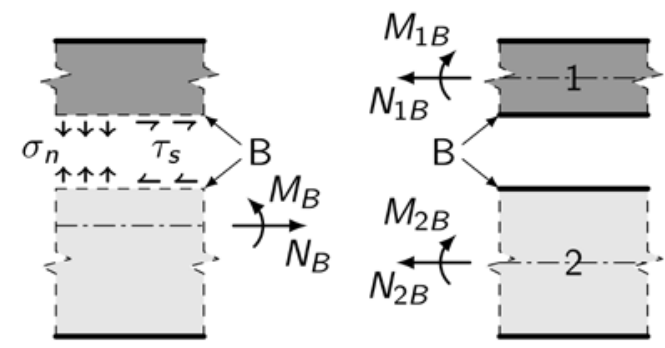

(b)

Fig. 1: A bimaterial DCB. (a) General description. (b) Interfacial stresses and crack tip forces. 

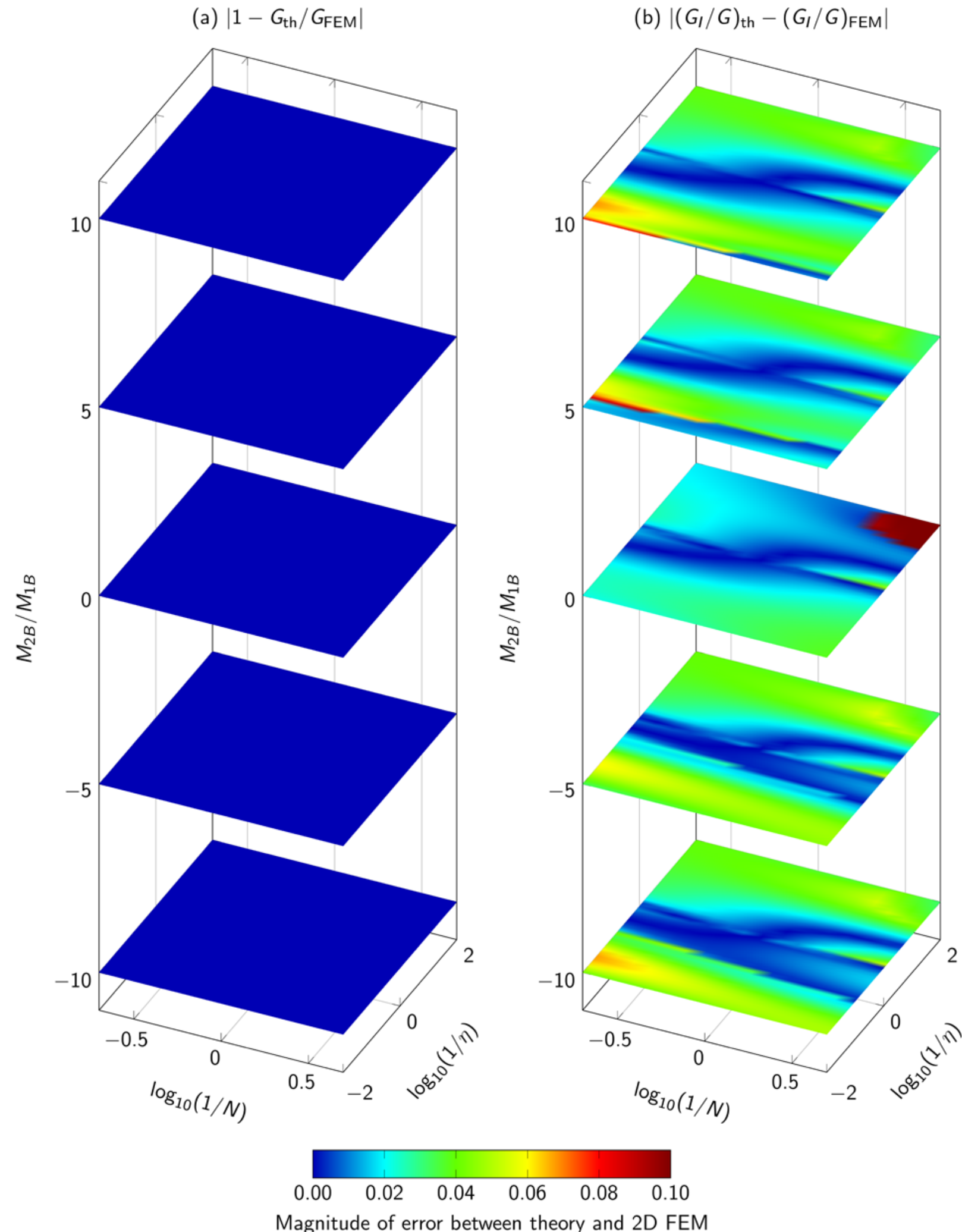

Fig. 2: Comparison of the present analytical theory and the 2D FEM for the total ERR $G$ and the ERR partition $G_{I} / G$ for variable $\eta, \mathrm{N}$ and $M_{2 B} / M_{1 B}$ with $\gamma=1$ and $\delta a=0.01$ under the plane stress condition. 

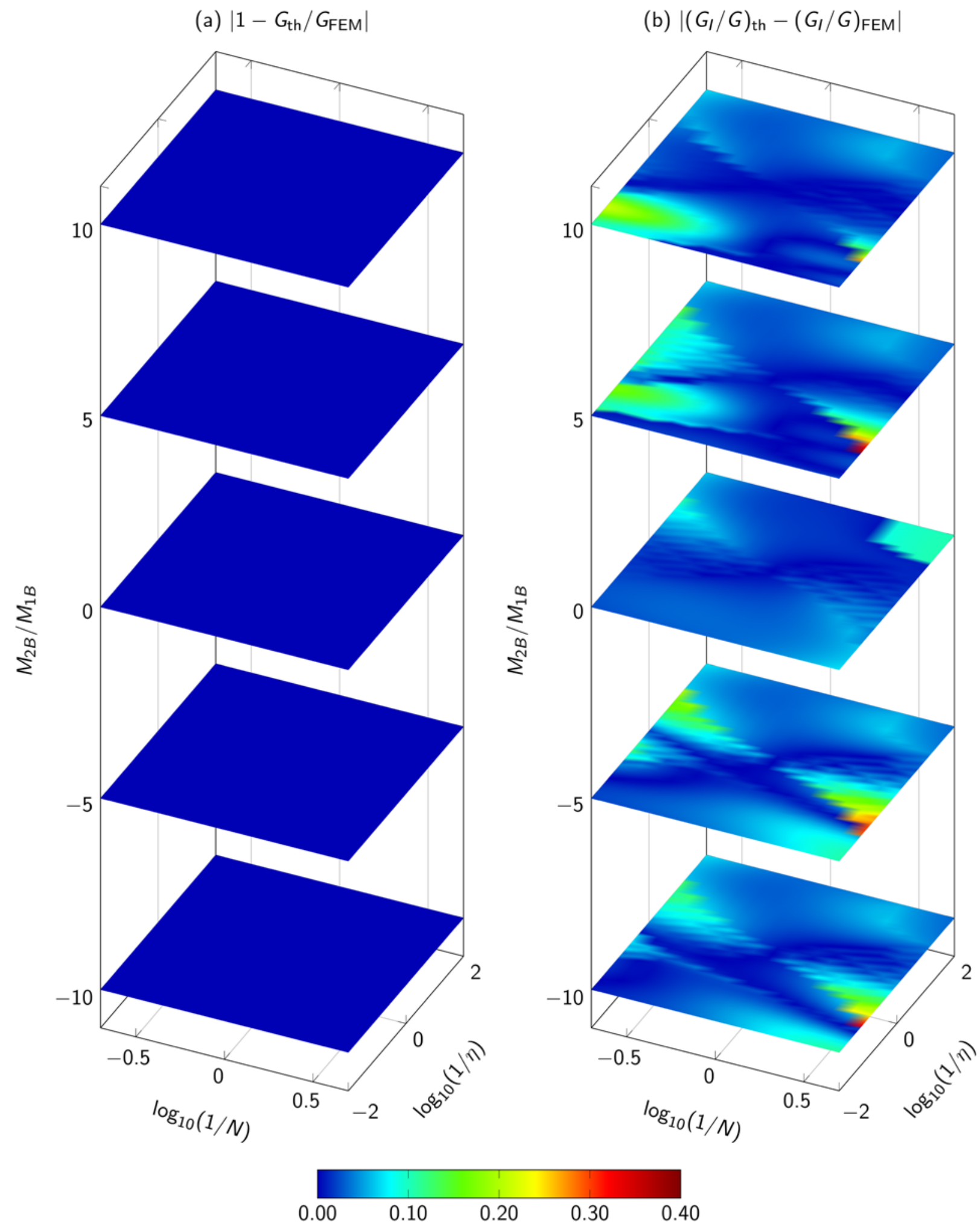

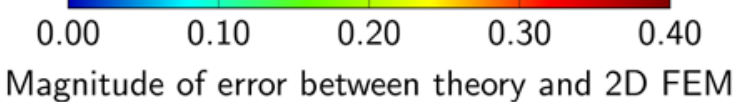

Fig. 3: Comparison of the present analytical theory and the 2D FEM for the total ERR $G$ and the ERR partition $G_{I} / G$ for variable $\eta, \mathrm{N}$ and $M_{2 B} / M_{1 B}$ with $\gamma=1$ and $\delta a=0.01$ under the plane strain condition. 

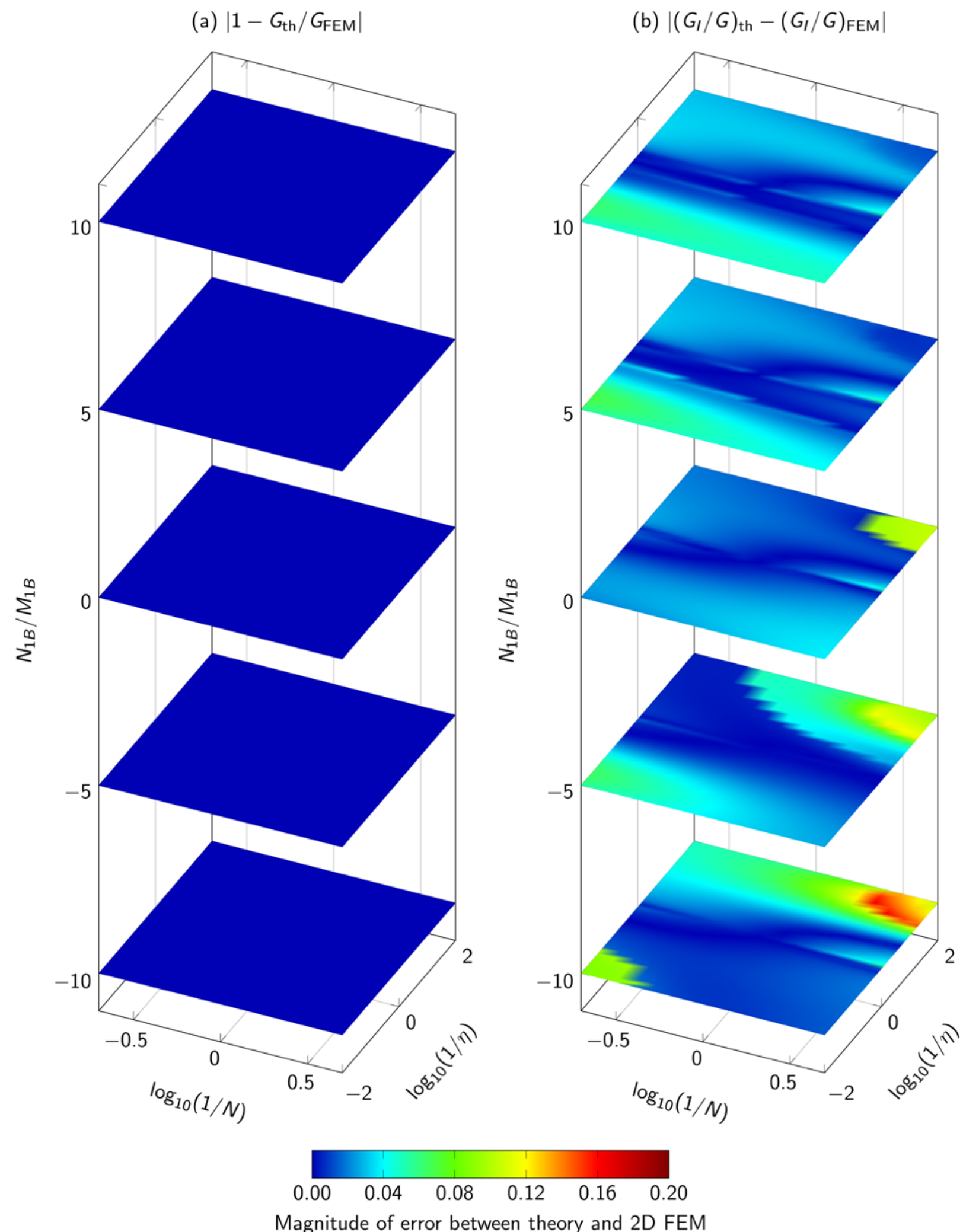

Fig. 4: Comparison of the present analytical theory and the 2D FEM for the total ERR $G$ and the ERR partition $G_{I} / G$ for variable $\eta, \mathrm{N}$ and $N_{1 B} / M_{1 B}$ with $\gamma=1$ and $\delta a=0.01$ under the plane stress condition. 

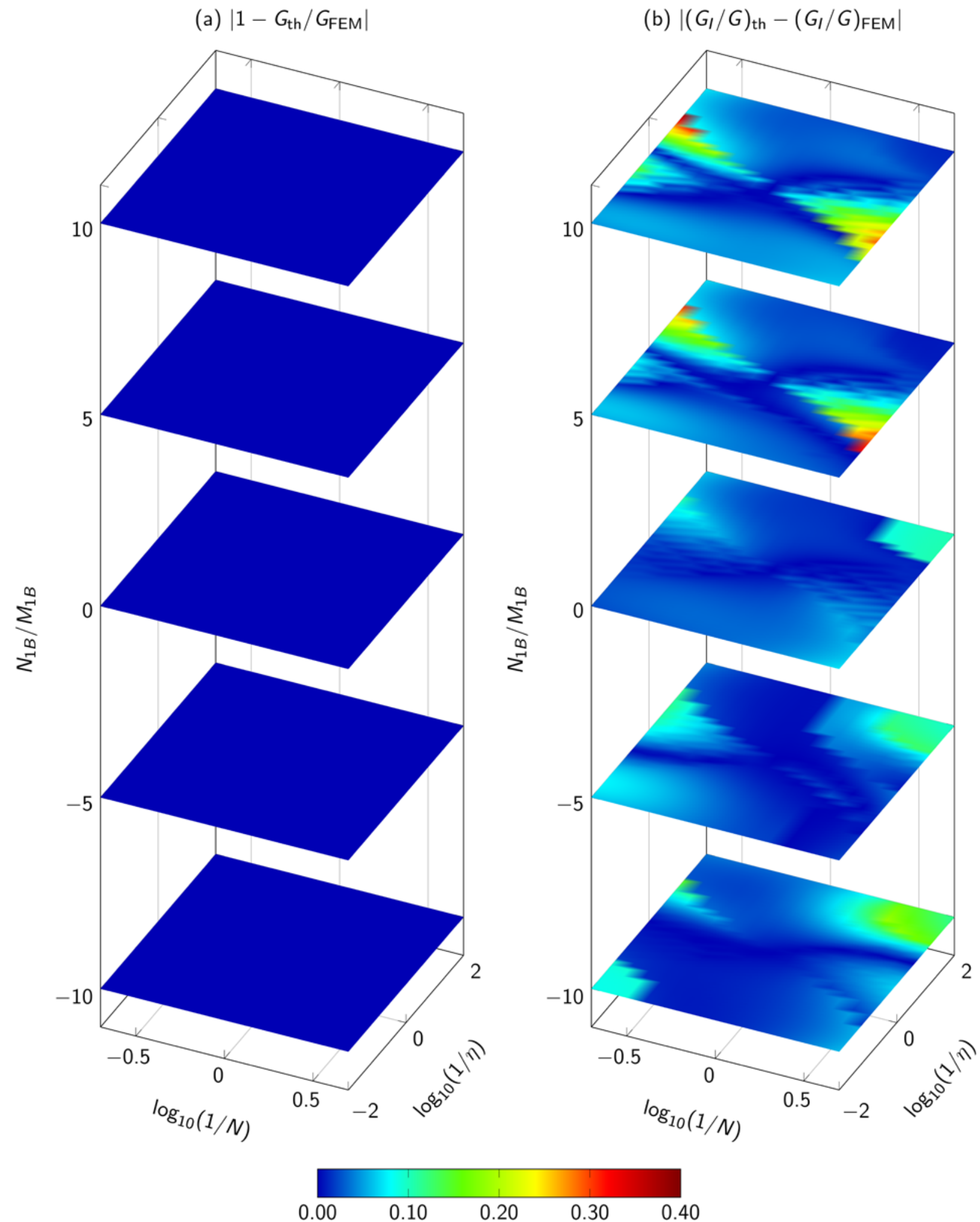

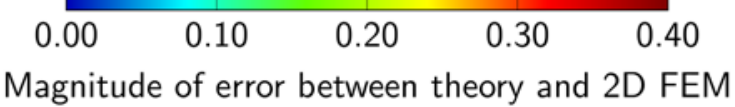

Fig. 5: Comparison of the present analytical theory and the 2D FEM for the total ERR $G$ and the ERR partition $G_{I} / G$ for variable $\eta, \mathrm{N}$ and $N_{1 B} / M_{1 B}$ with $\gamma=1$ and $\delta a=0.01$ under the plane strain condition. 